\title{
Guest editorial: Special section on Search-based Software Engineering track at GECCO 2018
}

Welcome to the special section of the Search-Based Software Engineering (SBSE) track from the 27th ACM Genetic and Evolutionary Computation Conference (GECCO), held in Kyoto, Japan, 15-19 July 2018.

The past two decades has witnessed a surge in the application of search-based and optimization techniques to a variety of software engineering tasks and problems. Applications range from test input data generation, project staffing, software refactoring, and optimization of requirements and design. The search-based software engineering community extends beyond the academic world, with many of the issues generated by software engineering professionals. This scenario results in high-quality practical research with strong industrial case studies. While still a relatively young field, the search-based software engineering community is thriving. Members of the community have produced a substantial body of work with important results.

The invited papers for this special section are revised and expanded versions of the full research papers accepted and presented at GECCO 2018. Based on GECCO reviews, presentations, and discussions during the conference, we invited the authors of four papers to extend their papers for this special section. The authors extended their papers by adding material to make new contributions to the literature. After review of the revised papers, we chose three for inclusion in this special section. The following provides a brief overview of the three included papers.

The first paper Standing on the Shoulders of Giants: Seeding Search-based Multi-Objective Optimization with Prior Knowledge for Software Service Composition by Tao Chen et al. deals with classical quality of service (QoS) optimization for service composition. Service composition and the resulting QoS is a challenging task due to often conflicting goals. Solutions that use multi-objective optimizations are promising approaches to handle the characteristics of these problems. Even though domain knowledge is often available, developers seldom, if ever, make use of that knowledge. This paper investigates how domain knowledge and domain knowledge seeding strategies could help tackle QoS service composition.

Simulation models are important in many domains. System creators often build models to simulate complex system behavior prior to building the actual system. For example, it is often not possible for developers to execute all available test cases for many cyber-physical systems. Therefore, simulation is an important tool. The second paper, Pareto efficient multi-objective black-box test case selection for simulation-based testing, by Arrieta et al., describes an approach that selects a sub-set of available tests cases by applying multiobjective optimization to black box testing information. The new approach performs significantly better than the traditional white box approach.

To save cost and development time, software teams often build complex software systems by reusing libraries. Developers access these libraries through their APIs. However, the lack of documentation for these APIs makes it difficult for human maintainers to understand the API constraints and usage patterns. The approach described in the third paper, Towards Assisting Developers in API Usage by Automated Recovery of Complex Temporal Patterns, by Saied et al., uses Genetic Programming to derive temporal usage patterns. These temporal usages patterns may reduce developers' program understanding effort and increase their productivity.

The papers in this special section should provide readers with insights into search-based software engineering. We thank all of the authors who submitted papers to the SBSE track of GECCO 2018, to the members of the program committee, and to additional reviewers for providing valuable feedback on the papers. Also, we thank the reviewers for the papers included in this special section. Their constructive feedback helped to shape the papers. Lastly, we thank Jeffrey Carver and Gunther Ruhe, editors of Information and Software Technology, for their support in developing this special section.

Giuliano Antoniol and Federica Sarro, Guest Editors 